\title{
ON THE STABILITY OF MULTIPLICATIVE INVERSE CUBIC FUNCTIONAL (MICF) EQUATION IN INTUTIONISTIC FUZZY NORMED SPACES
}

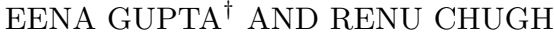

\author{
Date of Receiving : $\quad 26.02 .2020$ \\ Date of Revision : $\quad 07.05 .2020$ \\ Date of Acceptance : 08.05 .2020
}

\begin{abstract}
The objective of this study to examine the generalized Hyers- Ulam stability (GHUS) of multiplicative inverse cubic functional (MICF) equation of the form

$$
c(2 l+m)+c(l+2 m)=\frac{9 c(l) c(m)\left[c(l)+c(m)+2 c(l)^{\frac{1}{3}} c(m)^{\frac{1}{3}}\left[c(l)^{\frac{1}{3}}+c(m)^{\frac{1}{3}}\right]\right]}{\left[2 c(l)^{\frac{2}{3}}+2 c(m)^{\frac{2}{3}}+5 c(l)^{\frac{1}{3}} c(m)^{\frac{1}{3}}\right]^{3}}
$$

and using this, we give the Hyers stability, Hyers-Ulam-Rassias stability, UlamGavruta-Rassias product stability and JM Rassias mixed product-sum stability results of MICF equation in intutionistic fuzzy normed spaces (IFNS).
\end{abstract}

\section{Introduction}

Fuzzy set theory is a powerful hand set for modeling uncertainty and fuzziness in hetrogeneous problems emerging in the different fields of engineering and science. It has applications in many fields, for example, statistical convergence, chaos control, nonlinear dynamical systems, population dynamics, nonlinear operators, etc. ( [22], [30], [19]). The fuzzy topology proves to be a practical tool to deal with such circumstances where the use of classical theories ceases. The most interesting application of fuzzy topology in quantum particle physics arises in string and E-infinity theory of EI Naschie $([24],[25])$.

The impracticality of determining the position of particles gives the space-time a fuzzy structure[24]. Due to this fuzzy structure, position space representation of quantum mechanics ceases and therefore, a generalized normed space of quasi-position

2010 Mathematics Subject Classification. 39B82, 39B72.

Key words and phrases. Multiplicative inverse functional equation, intutionistic fuzzy normed space, generalized Hyers-Ulam stability, Ulam-Gavruta-Rassias stability.

The authors thanks the anonymous referees for their valuable comments as well as suggestions that helped to improve the presentation of this paper.

Communicated by: M. Mursaleem

${ }^{\dagger}$ Corresponding author. 